\title{
Impacto do ambulatório de reabilitação dermatocosmiátrica na qualidade de vida dos pacientes oncológicos da Faculdade de Medicina do ABC
}

\section{Impact of outpatient rehabilitation dermatocosmiatric on the quality of life of cancer patients at Faculdade de Medicina do ABC}

\author{
Dolores Gonzalez Fabra', Vanessa Salzano', Flávia Guglielnino', Ana Paula Kayo²
}

\begin{abstract}
Resumo
0 crescente interesse pelo conceito de qualidade de vida em saúde tem influenciado em muito as pesquisas científicas dos últimos anos, principalmente na área da Oncologia, em que não só o diagnóstico da doença como também o seu tratamento podem implicar importantes alterações psíquicas, físicas e sociais na vida dos pacientes e de seus familiares. 0 objetivo deste estudo foi avaliar o impacto do Ambulatório de Reabilitação Dermatocosmiátrica na qualidade de vida dos pacientes oncológicos da Faculdade de Medicina do ABC (FMABC). Por meio da aplicação de questionários individuais, observamos objetivamente uma melhora no grau de sofrimento, em torno de $82,5 \%$, e na qualidade de vida, variando de 50 a $100 \%$, quando procuramos tratar e reabilitar os pacientes para sua nova realidade, mesmo no decorrer do tratamento oncológico.
\end{abstract}

Palavras-chave: Dermatopatias; efeitos psicossociais da doença; qualidade de vida; questionários; reabilitação.

\section{Abstract}

The growing interest in the concept of quality of life in health has had much influence on the scientific research in recent years, especially in the area of Oncology, in which not only the diagnosis of the disease but also its treatment may imply important mental, physical and social changes in the life of these patients and their relatives. The aim of this study was to evaluate the impact of Dermatocosmiatric Rehabilitation Ambulatory of Faculdade de Medicina do $\mathrm{ABC}$ (FMABC) on the quality of life of cancer patients. By the means of the application of individual questionnaires, we objectively observed an improvement in the suffering degree, around $82.5 \%$, and in the quality of life, ranging from 50 to $100 \%$, when we aimed to treat and to rehabilitate the patients to their new reality, even during cancer treatment.

Keywords: Skin diseases; cost of illness; quality of life; questionnaires; rehabilitation.

Recebido: $28 / 2 / 2008$

Revisado: 9/11/2008

Aprovado: $31 / 1 / 2010$

2 Disciplina de Clínica Médica da FMABC Santo André (SP), Brasil

Endereço para correspondência: Dolores Gonzalez Fabra - Avenida Príncipe de Gales, 821 - Príncipe de Gales - CEP 09060-650 - Santo André (SP) -

E-mail: doloresgonzalezfabra@uol.com.br
} 


\section{Introdução}

Devido aos grandes avanços científicos nos tratamentos propostos aos pacientes oncológicos, ocorreu um aumento da expectativa de vida dos mesmos; porém, as consequências psicossociais cresceram de forma proporcional à maior sobrevida desses pacientes. Esse fato direcionou a atenção de diferentes especialidades para o estudo da qualidade de vida dos pacientes com tumores.

Esses estudos tratam da avaliação do impacto físico e psicossocial que a enfermidade ou as disfunções e incapacidades decorrentes dela ou de seu tratamento poderiam acarretar para as pessoas acometidas, permitindo, assim, um melhor conhecimento do paciente e sua adaptação a essa nova condição ${ }^{1}$.

O uso de questionários para mensurar a qualidade de vida é reconhecido como uma importante área científica na Saúde. Em controles clínicos experimentais, eles servem como instrumentos de medida dos resultados e são um importante componente de análise do custo-benefício do tratamento ${ }^{2}$. Dois tipos de medidas são utilizados para se mensurar a qualidade de vida: medidas genéricas e medidas relacionadas à saúde ou específicas a determinada doença. As medidas genéricas de qualidade de vida podem ser aplicadas a indivíduos saudáveis ou afetados por qualquer condição específica e focalizam as experiências e percepções subjetivas desses pacientes tanto em relação a padrões internos quanto a grupos externos de comparação. Já as medidas específicas procuram identificar os transtornos associados à doença ou às intervenções dela decorrentes e enfatizam os sintomas, funcionamentos e capacidades de cada paciente ${ }^{3}$.

Alguns estudos procuram relacionar a qualidade de vida de pacientes com câncer com um grupo controle, comprovando que mulheres com câncer de mama tiveram uma avaliação pior, principalmente em relação às disfunções do membro superior ipsilateral, e apresentaram piora da vida sexual ${ }^{4}$. Nesses estudos também foi encontrada uma piora da satisfação sexual de mulheres jovens com esse mesmo tipo de tumor quando comparadas aos seus respectivos grupos controles ${ }^{5,6}$; além disso, verificou-se que o diagnóstico de câncer de mama é mais traumático quanto mais jovem for a paciente e que as repercussões na sua imagem pessoal são mais depreciativas?

Outros autores avaliaram a qualidade de vida de cada grupo em específico, utilizando questionários aplicados a cada paciente. Por meio da aplicação de questionários, verificou-se que a depressão e a fadiga são os sintomas mais comuns que afetam a qualidade de vida de pacientes com câncer de pulmão ${ }^{8,9}$ e também de mulheres com câncer uterino submetidas à histerectomia, sendo a depressão o principal fator de infelicidade e desesperança, o que leva a uma diminuição dos interesses pelas atividades cotidianas, perda ou ganho de peso, insônia, fadiga e ideias suicidas ${ }^{10}$. Comparando-se os pacientes com câncer retal em relação ao sexo, foi avaliado que a insatisfação com a imagem corporal não teve diferenças entre os sexos e que as mulheres relataram uma diminuição do seu poder de atração e o homem, da sua virilidade ${ }^{11}$. Em pacientes tratadas de cân- cer de mama, comprovou-se que esse tumor tem um grande impacto no papel social dessas mulheres (na família, no trabalho, na sexualidade) (2,13 $^{12}$ Nesse grupo de pacientes, quanto melhor o nível socioeconômico e de escolaridade (além de relação marital estável e cirurgia com conservação mamária), melhores são as taxas de qualidade de vida ${ }^{14}$.

A interferência do tumor e de seu tratamento na qualidade de vida dos pacientes oncológicos estimulou a formação de grupos de apoio, visando unificar e fortalecer esses pacientes, dando-lhes respostas objetivas a suas dúvidas e reintegrando-os novamente à sociedade. Os grupos de apoio para pacientes com câncer ovariano são uma grande oportunidade de troca de experiências, informações e emoções, o que contribui sobremaneira para uma melhora da sua qualidade de vida ${ }^{15}$. Comparando-se pacientes com câncer inclusos em grupos de apoio e pacientes com câncer que não participaram dessa inclusão, foi comprovado que os pacientes do primeiro grupo aprenderam a lidar melhor com a doença e seu tratamento, portanto, não apresentaram piora significativa de sua qualidade de vida ${ }^{16}$.

Foi com esse objetivo que instituímos o Ambulatório de Reabilitação Dermatocosmiátrica da Faculdade de Medicina do ABC (FMABC). Trata-se de um projeto pioneiro de assistência integral aos pacientes oncológicos, realizado em conjunto entre as disciplinas de Dermatologia e Oncologia da FMABC, além da colaboração de outras especialidades, tais como Cirurgia Plástica, Psiquiatria, Fisioterapia, entre outras.

Os pacientes oncológicos que foram submetidos a tratamento quimio/radioterápico e/ou cirúrgico na Faculdade de Medicina do ABC passaram previamente pelo Ambulatório de Reabilitação, onde receberam orientações preventivas dermatocosmiátricas a fim de serem minimizados os efeitos colaterais e as sequelas do tratamento oncológico. Tendo sido iniciado o tratamento proposto para cada paciente, o mesmo é reavaliado quinzenalmente para acompanhamento e tratamento dos possíveis efeitos colaterais dermatocosmiátricos decorrentes da quimioterapia e/ou radioterapia e/ou cirurgia.

Com o suporte e o acompanhamento dermatológicos do paciente oncológico nesse ambulatório multidisciplinar, visamos promover um maior equilíbrio estético e emocional antes, durante e após o tratamento oncológico, melhorando sua autoestima e sua qualidade de vida, reintegrando-o ao seu ambiente familiar, social e ocupacional, além de estimular sua vontade de viver.

O objetivo deste trabalho foi avaliar o impacto do Ambulatório de Reabilitação Dermatocosmiátrica da FMABC na qualidade de vida dos pacientes oncológicos, além de catalogar as alterações dermatológicas decorrentes do tratamento oncológico - quimio e/ou radioterápico e/ ou cirúrgico.

\section{Casuística e métodos}

Para avaliar o impacto do Ambulatório de Realibitação Dermatocosmiátrica da FMABC na qualidade de vida dos pacientes oncológicos, foi aplicado o questionário de Índice de Qualidade de Vida em 
Dermatologia (DLQI, do inglês Dermatology Life Quality Index), com autorização da sua utilização pelo Prof. Dr. Joy Hayes, do Departamento de Dermatologia da Wales College of Medicine - Cardiff University. Esse questionário, validado e traduzido para o português ${ }^{17}$, aborda sinais e sintomas da dermatose, atividades diárias, lazer, vida profissional/escolar, relacionamentos pessoais e tratamentos. Seu índice máximo é de 30 pontos, o que refletiria sofrimento extremo (incompatível com a vida), e o índice mínimo é de 0 pontos, o que refletiria ausência de sofrimento. A pontuação foi graduada da seguinte forma:

0-1: sofrimento que não afeta a vida;

2-5: sofrimento que afeta pouco a vida;

6-10: sofrimento que afeta moderadamente a vida;

11-20: sofrimento que afeta muito a vida;

21-30: sofrimento que afeta extremamente a vida.

Instituímos o Ambulatório a partir de novembro de 2004, para agendamento no ano corrente de 2005, e iniciamos o atendimento à população geral apenas a partir de janeiro de 2005. Foram atendidos 229 pacientes no período de janeiro de 2005 a junho de 2006, tendo sido realizados 765 atendimentos entre consultas e retornos dos mesmos.

Para a aplicação do questionário, foram selecionados 61 pacientes de forma aleatória, com exclusão prévia das crianças menores de 16 anos, para maior fidedignidade estatística. O questionário foi aplicado imediatamente antes do acompanhamento no Ambulatório de Reabilitação e no final do acompanhamento, sendo, então, comparados para a avaliação das alterações na qualidade de vida desses pacientes, mensurando o grau de sofrimento. Avaliamos alterações na aparência física e o surgimento de lesões dermatológicas decorrentes do tratamento oncológico.

Além desse questionário, os pacientes selecionados responderam, ao final do acompanhamento, à seguinte pergunta: "Em quantos por cento o Ambulatório de Reabilitação Dermatocosmiátrica melhorou sua qualidade de vida durante o tratamento oncológico?” Essa questão avaliou a melhora na qualidade de vida de forma subjetiva.

Foi feita a análise estatística descritiva de todas as variáveis do estudo. As variáveis qualitativas foram apresentadas em termos de seus valores absolutos e relativos. As variáveis quantitativas foram apresentadas em termos de seus valores de tendência central e de dispersão. Para se comparar o valor inicial e final, foi utilizado o teste de Wilcoxon. O nível de significância foi de 5\%. O pacote estatístico utilizado foi o Statistical Package for Social Sciences (SPSS), versão 14.0 para Windows.

Este estudo foi aprovado pelo Comitê de Ética e Pesquisa da FMABC, protocolo n ${ }^{\circ}$ 053/2008.

\section{Resultados}

Nos questionários aplicados, foi observada, de forma objetiva, uma melhora no grau de sofrimento do paciente entre 30 e 100\%, com uma média de $85,2 \%$. Visando-se a uma avaliação subjetiva dos pacientes, os mesmos responderam à pergunta final: "Em quantos por cento o Ambulatório de Reabilitação Dermatocosmiátrica melhorou sua quali- dade de vida durante o tratamento oncológico?" As respostas variaram de 50 a 100\% de melhora da sua qualidade de vida (Figura 1).

Apesar de o Ambulatório ser aberto para pacientes de qualquer sexo, idade, tipo de tumor diagnosticado e tratamento oncológico proposto, a grande maioria dos pacientes oncológicos atendidos em nosso ambulatório era composta por mulheres (86\%), sendo a manifestação tumoral mais frequente o carcinoma de mama - mais de $68 \%$ dos casos (Tabela 1) - e o tratamento oncológico proposto com maior frequência a esses pacientes foi a exérese tumoral associada à quimio e à radioterapia (Tabela 2).

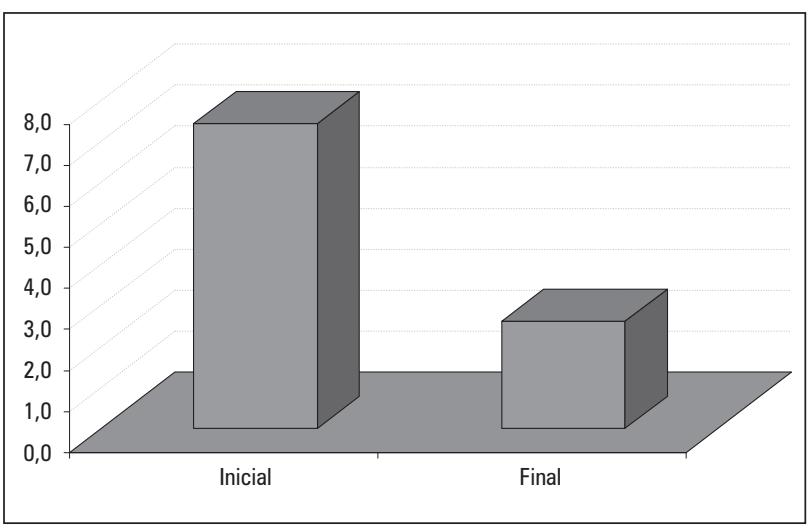

Figura 1 - Avaliação descritiva para a qualidade de vida nos momentos inicial e final.

Tabela 1 - Classificação dos tumores dos pacientes atendidos no Ambulatório de Reabilitação Dermatocosmiátrica da Faculdade de Medicina do ABC

\begin{tabular}{lcc}
\hline Tipo de tumor & $\begin{array}{c}\text { Valor } \\
\text { absoluto }\end{array}$ & $\begin{array}{c}\text { Valor } \\
\text { relativo }\end{array}$ \\
\hline Carcinoma de mama & 156 & 0,681 \\
Carcinoma de mama com metástase hepática & 4 & 0,017 \\
Carcinoma de mama com metástase óssea & 7 & 0,03 \\
Adenocarcinoma de próstata & 7 & 0,03 \\
Carcinoma espinocelular de reto & 5 & 0,021 \\
Linfoma de Hodgkin & 6 & 0,026 \\
Linfoma não-Hodgkin & 9 & 0,039 \\
Adenocarcinoma de cólon & 6 & 0,026 \\
Carcinoma espinocelular de laringe & 8 & 0,034 \\
Carcinoma espinocelular de esôfago & 5 & 0,021 \\
Outros & 16 & 0,069 \\
Valor total & 229 & 1 \\
\hline
\end{tabular}

Tabela 2 - Tratamentos oncológicos realizados independentemente do tipo de tumor

\begin{tabular}{lcc}
\hline Tipo de tratamento oncológico & Valor absoluto & Valor relativo \\
\hline Exérese tumoral & 20 & 0,087 \\
Exérese e OT & 39 & 0,126 \\
Exérese e RT & 22 & 0,096 \\
OT e RT & 19 & 0,082 \\
OT & 18 & 0,078 \\
RT & 6 & 0,026 \\
Exérese, OT e RT & 105 & 0,458 \\
Valor total & 229 & 1 \\
\hline
\end{tabular}

OT: quimioterapia; RT: radioterapia. 
Os tumores classificados como "outros", na Tabela 2, apresentaram apenas um caso dentre todos os pacientes atendidos, sendo:

linfoma de Burkitt;

carcinoma pulmonar de pequenas células;

carcinoma espinocelular da boca;

cistoadenocarcinoma do pâncreas;

adenocarcinoma do cólon com metástase hepática;

carcinoma da língua;

adenocarcinoma da próstata com metástase para coluna vertebral; adenocarcinoma da próstata associado a carcinoma espinocelular da face;

carcinoma espinocelular das cordas vocais;

carcinoma da faringe;

rabdomiossarcoma;

adenocarcinoma papilífero bilateral do ovário;

adenocarcinoma do sigmoide;

carcinoma do testículo;

melanoma;

carcinoma papilífero não encapsulado da tireoide.

\section{Discussão}

De acordo com a Organização Mundial de Saúde (OMS) ${ }^{18}$, a definição de qualidade de vida é "a percepção do indivíduo de sua posição na vida, no contexto da cultura e sistemas de valores nos quais ele vive e em relação aos seus objetivos, expectativas, padrões e preocupações". O conceito de qualidade de vida é multidimensional e inclui a saúde física e mental do paciente, seu estado psicológico, seu nível de independência, seu bem-estar social e sua relação com o meio ambiente ${ }^{19}$, sendo avaliada do ponto de vista do próprio paciente $^{20}$.

Sabemos que as lesões cutâneas podem causar um forte impacto social, emocional, familiar e profissional, diminuindo a autoes-

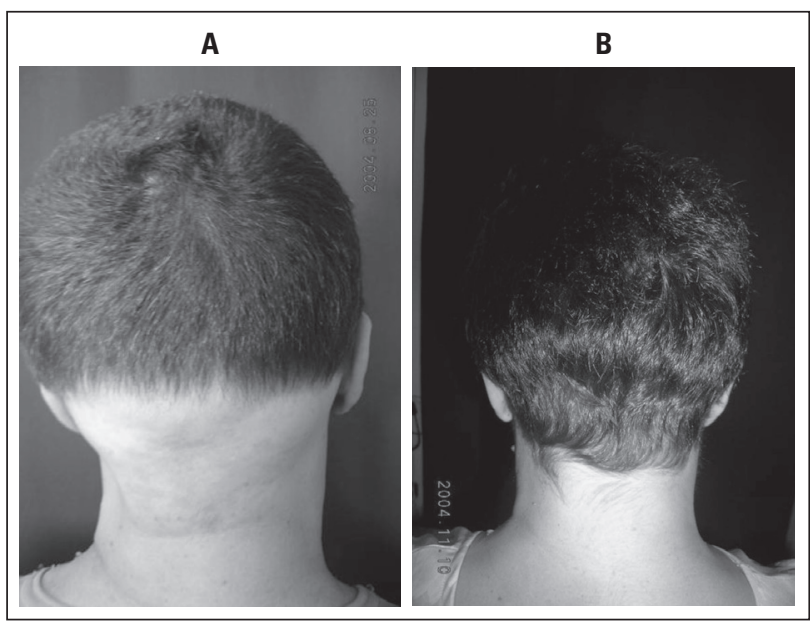

Figura 2 - Depilação da região occipital em paciente com câncer de tireoide durante a radioterapia. (A) Antes da reabilitação; (B) após reabilitação. tima e a qualidade de vida do paciente, causando constrangimento e isolamento - situações que favorecem ou intensificam quadros depressivos.

Os estudos de qualidade de vida em Dermatologia utilizam índices e escalas para interpretarem muitos dos questionamentos realizados com os pacientes, da maneira mais objetiva possível, visando incluir seus sintomas, desconforto físico, suas limitações (físicas e psicológicas), os efeitos cognitivos apresentados, alterações de suas atividades diárias (profissionais, familiares, sexuais), os efeitos sociais, além de seus medos ${ }^{21,22}$.

$\mathrm{Na}$ grande maioria dos casos, a aparência física e o desconforto dermatológico decorrente do tratamento quimio e/ou radioterápico e/ ou cirúrgico transforma-se em um estigma, pois geralmente o paciente oncológico pode manter suas atividades sociais e profissionais e não o faz por vergonha da sua aparência, preferindo manter-se fechado em casa. Isso passa a ser um forte fator de exclusão social, que mantém esse paciente isolado e alheio à vida, acentuando ou desencadeando quadros depressivos, diminuindo sua imunidade e dificultando a resposta terapêutica e a adesão ao tratamento oncológico.

Os fatores associados à satisfação dos pacientes em tratamento dermatológico incluem a capacidade do especialista em explicar o tipo de tratamento proposto, seus efeitos e reações e o respeito pelos anseios do paciente por parte do profissional, mostrando empatia por ele e por sua condição, aprimorando, portanto, a relação médico-paciente $^{23,24}$. Em nosso ambulatório, notamos que a explicação detalhada da dermatopatia e da evolução cosmiátrica do tratamento aumentou consideravelmente a adesão do paciente aos procedimentos, como, por exemplo, o tipo de repilação dos fios de cabelo, as características quanto à forma e coloração dos mesmos (Figura 2), a possibilidade de perda dos pêlos pubianos, alterações das lâminas ungueais (Figura 3), entre outros.

Nosso trabalho mostrou um impacto extremamente positivo na qualidade de vida dos pacientes acompanhados em nosso ambulatório, pois preparamos seu lado emocional para aquilo que estava por vir em termos de aparência, e os orientamos acerca de como deveriam lidar com essas novas situações, reforçando sempre que essas alterações dermatológicas e cosmiátricas são transitórias e passíveis de tratamento. Além disso, enfatizamos que, durante o tratamento quimio e radioterápico, existem mecanismos de camuflagem que reintegram o paciente à sua vida cotidiana, social, profissional e familiar.

A abordagem dos pacientes submetidos a tratamentos oncológicos exige um acompanhamento multidisciplinar ${ }^{25}$, incluindo também um suporte emocional antes, durante e depois do tratamento ${ }^{26}$, objetivos pelos quais foi criado o Ambulatório de Reabilitação Dermatocosmiátrica da FMABC, onde essa integração tornou-se realidade. Antes da criação desse ambulatório, quando qualquer paciente em tratamento quimio e/ou radioterápico procurava um dermatologista para minimizar essas alterações de pele, era orientado a, previamente, terminar o tratamento oncológico para só posteriormente solucionar as sequelas 


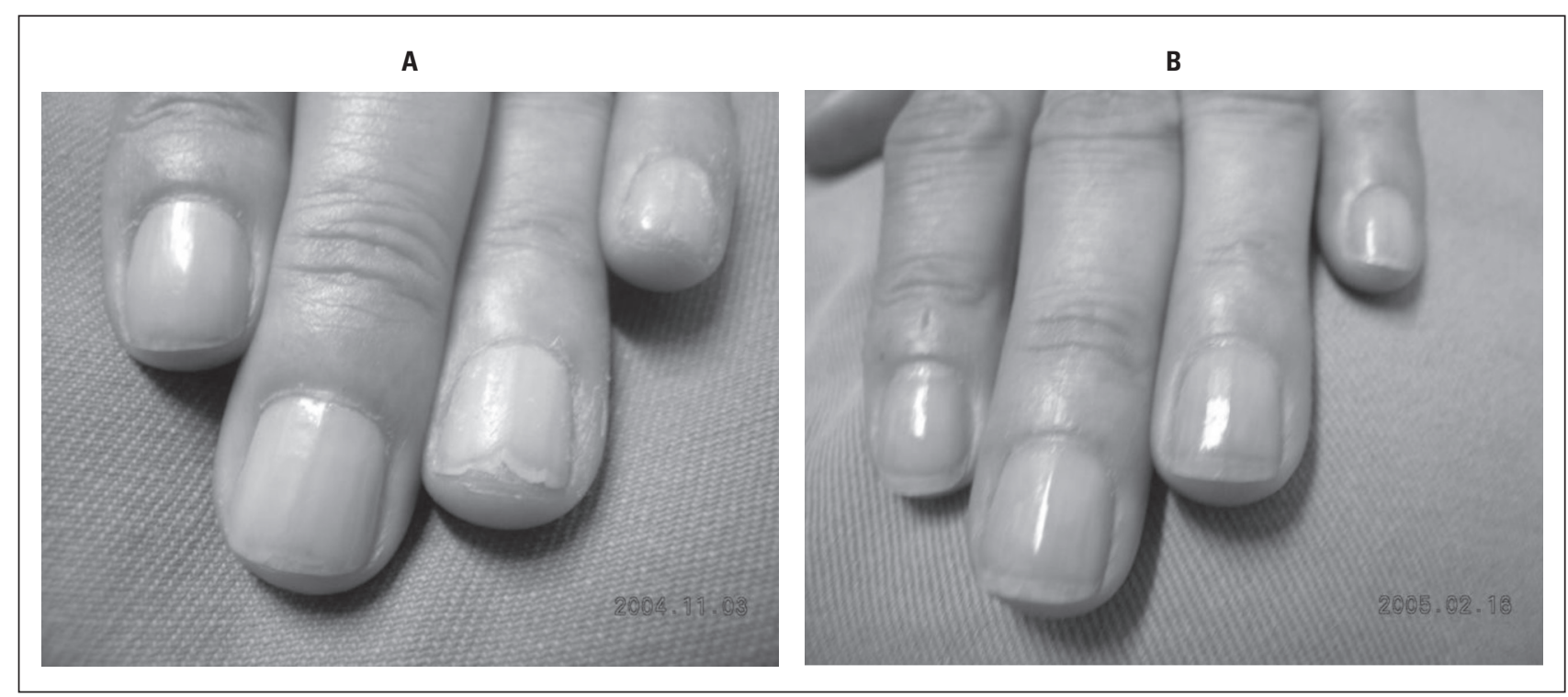

Figura 3 - Regressão da onicólise mesmo durante as sessões de quimioterapia. (A) Antes da reabilitação; (B) após reabilitação.

dermatocosmiátricas. Porém, é principalmente nessa fase que o paciente mais precisa de auxílio.

Observamos objetivamente uma melhora no grau de sofrimento do paciente em torno de $85,2 \%$ e subjetivamente uma melhora na qualidade de vida entre 50 a $100 \%$. Portanto, o impacto do Ambulatório de Reabilitação Dermatocosmiátrica da FMABC foi extremamente favorável, pois apresentou significância estatística com $\mathrm{p}<0,001$. Nesse ambulatório, conseguirmos catalogar, de forma precisa, as alterações dermatológicas decorrentes do tratamento oncológico - quimio e/ ou radioterápico e/ou cirúrgico - e tratá-las durante o mesmo, sem interferir no procedimento proposto.

\section{Agradecimentos}

Ao Professor Auro Del Giglio, da Disciplina de Oncologia da FMABC e aos Professores Carlos D’Apparecida Santos Machado Filho, Luiz Henrique de Camargo Paschoal e Francisco Paschoal, da Disciplina de Dermatologia da FMABC.

\section{Referências}

1. Seidl EMF, Zannon CMLC. Qualidade de vida e saúde: aspectos conceituais e metodológicos. Cad Saúde Publica. 2004;20(2):580-8.

2. Carr AJ, Thompson PW, Kirwan JR. Quality of life measures. $\mathrm{Br} \mathrm{J}$ Rheumatol. 1996;35(3):275-81.

3. Prebianchi HB. Medidas de qualidade de vida para crianças: aspectos conceituais e metodológicos. Psicol Teor Prat. 2003;5(1):57-69.

4. Dorval M, Maunsell E, Deschênes L, Brisson J, Mâsse B. Long-term quality of life after breast cancer: comparison of 8-year survivors with population controls. J Clin Oncol. 1998;16(2):487-94.

5. Ganz PA, Rowland JH, Desmond K, Meyerowitz BE, Wyatt GE. Life after breast cancer: understanding women's health-related quality of life and sexual functioning. J Clin Oncol. 1998;16(2):501-14.

6. Ganz PA, Coscarelli A, Fred C, Kahn B, Polinsky M, Petersen L. Breast cancer survivors: psychosocial concerns and quality of life. Breast Cancer Res Treat. 1996;38(2):183-99.

7. Bakewell RT, Volker DL. Sexual dysfunction related to the treatment of young women with breast cancer. Clin J Oncol Nurs. 2005;9(6):697-702.
8. Fox SW, Lyon DE. Symptom clusters and quality of life in survivors of lung cancer. Oncol Nurs Forum. 2006;33(5):931-6.

9. Rolke HB, Bakke PS, Gallefoss F. Heath related quality of life, mood disorders and coping abilities in an unselected sample of patients with primary lung cancer. Respir Med. 2008;102(10):1460-7.

10. Vomvolaki E, Kalmantis K, Kioses E, Antsaklis A. The effect of hysterectomy on sexuality and psychological changes. Eur J Contracept Reprod Health Care. 2006;11(1):23-7.

11. Burghofer K, Jauch KW. Sexuality and body image in patients with rectal cancer. MMW Fortschr Med. 2006;148(18):36-7.

12. Ferrel RB, Grant M, Funk B, Otis-Green S, Garcia N. Quality of life in breast cancer. Part I: Physical and social well-being. Cancer Nurs. 1997;20(6): 398-408.

13. Ferrel RB, Grant M, Funk B, Otis-Green S, Garcia N. Quality of life in breast cancer. Part II: Psychological and spiritual well-being. Cancer Nurs. 1998;21(1):1-9.

14. Huguet PR, Morais SS, Osis MJD, Pinto-Neto AM, Gurgel MSC. Qualidade de vida e sexualidade de mulheres tratadas de câncer de mama. Rev Bras Ginecol Obstet. 2009;31(2):61-7. 
15. Ahlberg K, Nordner A. The importance of participation in support groups for women with ovarian cancer. Oncol Nurs Forum. 2006;33(4):E53-61.

16. Grande GE, Myers LB, Sutton SR. How do patients who participate in cancer support groups differ from those who do not? Psychooncology. 2006:15(4):321-34.

17. Ferraz LB, Almeida FA, Vasconcellos MR, Faccina AS, Ciconelli RM, Ferraz $M B$. The impact of lupus erythematosus cutaneous on the Quality of life: the Brazilian-Portuguese version of DLOI. Qual Life Res. 2006;15(3):565-70.

18. [No authors listed]. The World Health Organization Quality of Life assessment (WHOQOL): position paper from the World Health Organization. Soc Sci Med. 1995;41(10):1403-9.

19. Fortune DG, Main CJ, O'Sullivan TM, Griffiths CE. Quality of life in patients with psoriasis: the contribution of clinical variables and psoriasis-specific stress. Br J Dermatol. 1997;137(5):755-60.

20. Cella DF, Cherin EA. Quality of life during and after cancer treatment. Compr Ther. 1988;14(5):69-75.
21. Halioua B, Beumont MG, Lunel F. Quality of life in dermatology. Int $J$ Dermatol. 2000;39(11):801-6

22. Finlay AY. Quality of life measurement in dermatology: a practical guide. Br J Dermatol. 1997;136(3):305-14.

23. Renzi C, Abeni D, Picardi A, Agostini E, Melchi CF, Pasquini P et al. Factors associated with patient satisfaction with care among dermatological outpatients. Br J Dermatol. 2001;145(4):617-23.

24. Renzi C, Tabolli S, Picardi A, Abeni D, Puddu P, Braga M. Effects of patient satisfaction with care on health-related quality of life: a prospective study J Eur Acad Dermatol Venereol. 2005;19(6):712-8.

25. Couceiro TCM, Menezes TC, Valênça MM. Síndrome dolorosa pósmastectomia: a magnitude do problema (revisão). Rev Bras Anestesiol. 2009;59(3):358-65.

26. Sampaio EBM, Albuquerque IMN, Linhares JJ. Assessing the quality of life in women with breast cancer through the questionnaire SF-36. Appl Cancer Res. 2008;28(3):93-8. 